\title{
Desenvolvimento de Experimentos Sobre Comunicação Digital Empregando Tecnologia FPGA para Laboratório de Ensino de Telecomunicações
}

\author{
Allan Borgato*, Marina Q. Tavares, Michel D. Yacoub e Paulo Cardieri.
}

\section{Resumo}

O projeto consistiu na construção de uma plataforma de trabalho e na preparação de experimentos sobre comunicação digital, utilizando a técnica de geração de sinais Síntese Digital Direta (DDS) para implementar sistemas de modulação digital em uma plataforma de desenvolvimento de Arranjo de Portas Programáveis em Campo (FPGA). A técnica DDS consiste no uso de uma tabela que armazena amostras de um período da forma de onda desejada (senoidal, por exemplo) e um contador para ajustar a fase do sinal gerado. A plataforma desenvolvido poderá ser a base para diversos experimentos envolvendo comunicações digitais.

\section{Palavras-chave:}

Comunicação digital, ensino de técnicas de modulação, FPGA.

\section{Introdução}

Atualmente a maioria dos dispositivos eletrônicos processa informações digitalmente, devido a flexibilidade para reconfigurações e usufruto dos avanços constantes nos campos de microeletrônica e processamento. Os processos de modulação e demodulação digital, apesar de envolverem, em última análise, sinais analógicos, podem ser implementados por meio de processamento digital de sinais.

Aliando esse cenário ao fato de que a Faculdade de Engenharia Elétrica e de Computação da UNICAMP possui plataformas de desenvolvimento com tecnologia FPGA em seu inventário, desenvolveu-se nesse projeto uma plataforma de trabalho para a implementação de moduladores e demoduladores digitais. Tal base poderá ser usada em experiências em laboratório nas disciplinas de graduação, possibilitando ao aluno conhecer as técnicas de geração de sinais analógicos por meio de processamento de sinais, além de investigar as características de algumas técnicas de modulação digital.

\section{Resultados e Discussão}

Empregando a plataforma FPGA Altera Cyclone II, foi desenvolvido um sistema baseado na técnica Síntese Digital Direta (DDS ${ }^{1}$ ) que sintetiza as formas de onda de quatro técnicas de modulação digital, a saber:

1) BPSK (Modulação binário por fase): Varia a fase da portadora de acordo com o valor de um bit.

2) FSK (Modulação por frequência): Varia a frequência da portadora de acordo com o valor de um bit.

3) QPSK (Modulação por fase em quadratura): Varia a fase da portadora de acordo com o valor de dois bits.

4) 16QAM (Modulação por amplitude em quadratura): Varia a amplitude $e$ fase da portadora de acordo com o valor de quatro bits.

O sistema conta com fácil utilização por parte do usuário, que poderá selecionar, através de chaves presentes na plataforma FPGA, (I) a informação digital a ser modulada, (ii) a frequência da portadora, (iii) a quantidade de ciclos de portadora por símbolo transmitido e (iv) a técnica de modulação, dentre as citadas acima.
Tal implementação foi preparada para potenciais experimentos a serem adotados no laboratório de ensino de comunicações, de forma que, com a utilização de equipamentos secundários existentes no laboratório, como osciloscópio e analisador de espectro, o aluno poderá estudar algumas características das modulações digitais, como ocupação do espectro, relação entre banda ocupada e taxa de bits ou símbolos transmitidos, e avaliar o desempenho de cada tipo de modulação quanto à taxa transmissão de dados e aos efeitos de ruídos.

Como prosseguimento desse projeto e visando a adoção essa plataforma nas disciplinas de laboratório, será desenvolvido um subsistema que receba uma sequência de bits (gerados a partir de uma fonte externa de sinais) e introduza-a no circuito modulador, gerando a sequência de símbolos modulados. Além disso, será elaborado um roteiro dos experimentos, com uma breve explicação sobre a técnica de geração de sinais DDS, expondo algumas partes específicas dos códigos referentes aos blocos lógicos que constituem o sistema, escritos em linguagem VHDL. Dessa forma, o aluno poderá ter uma maior compreensão de como é possível construir um sistema de modulação digital, além de sugerir ao aluno alterações no código e/ou lógica para implementação de outras técnicas de modulação.

\section{Conclusões}

Graças à versatilidade do sistema de modulação digital desenvolvido, a plataforma de trabalho poderá ser usada como base para a implantação de diferentes experimentos nos laboratórios de ensino de comunicações da FEEC, constituindo numa valiosa ferramenta de ensino.

\section{Agradecimentos}

Ao CNPq, instituição que fomentou o projeto através do PIBIC; à UNICAMP, que realiza o programa em conjunto com o CNPq.

\footnotetext{
${ }^{1}$ MURPhY, E.; SLATTERY, C. Ask The Application Engineer-33: All About Direct Digital Synthesis. Analog Dialogue, Massachusetts, EUA, v. 38, ago. 2008. Disponível em <http://www.analog.com/en/analogdialogue/articles/all-about-direct-digital-synthesis.html>. Acesso em: 10 ago. 2018
} 